\title{
Models of a post-combustion absorption unit for simulation, optimiza- tion and non-linear model predictive control schemes
}

\author{
J. Åkesson ${ }^{\mathrm{a}, \mathrm{d}}$, R. Faber ${ }^{\mathrm{b}}$, C. D. Laird ${ }^{\mathrm{c}}$, K. Prölß ${ }^{\mathrm{a}}$, H. Tummescheit ${ }^{\mathrm{a}}$, S. Velut ${ }^{\mathrm{a}}$, Y. Zhu $^{\mathrm{c}}$ \\ ${ }^{a}$ Modelon AB, Ideon Science Park, Lund, Sweden \\ ${ }^{\mathrm{b}}$ Vattenfall Research and Development AB, Berlin, Germany \\ ${ }^{c}$ Artie McFerrin Department of Chemical Engineering, Texas A\&M University, U.S.A \\ ${ }^{\mathrm{d}}$ Department of Automatic Control, Lund University, Sweden
}

\begin{abstract}
An increasing demand on load flexibility in power supply networks is the motivation to look at flexible, and possibly optimal control systems for power plants with carbon capture units. Minimizing the energy demand for carbon dioxide removal under these circumstances reduces the cost disadvantage of carbon capture compared to conventional production. In this work a dynamic model in Modelica of a chemical absorption process run with an aqueous monoethanolamine (MEA) is developed, and used for solving optimal control problems. Starting from a rather detailed dynamic model of the process, model reduction is performed based on physical insight. The reduced model computes distinctly faster, shows similar transient behavior and reflects trends for optimal steady-state operations reported in the literature. The detailed model has been developed in Dymola, and the reduced model is used in JModelica.org, a platform supporting non-linear dynamic optimization. First results are shown on the dynamic optimization of the desorption column, the main cause of energy usage in the process.
\end{abstract}

Keywords: $\mathrm{CO}_{2}$, absorption, model, optimization, nonlinear model predictive control, Modelica, JModelica,org

\section{Introduction}

Carbon dioxide $\left(\mathrm{CO}_{2}\right)$ removal from a gas mixture using aqueous amine solutions is a well established process that previously has mainly been applied to gas sweetening of natural gas in refineries. Although the focus there lies primarily on the removal of hydrogen sulfide, it is equally applicable to flue gas from fossil-fuel fired power plants.

Figure 1 shows a schematic of the process. The $\mathrm{CO}_{2}$ from the flue gas is absorbed by the liquid solvent in the absorber column. The cleaned gas is re- leased to the environment, while the rich solution is pumped to the stripper column passing through a heat exchanger on the way. In the stripper at elevated temperatures, the $\mathrm{CO}_{2}$ in the solution is released to a steam flow from the reboiler, which is driven by bled

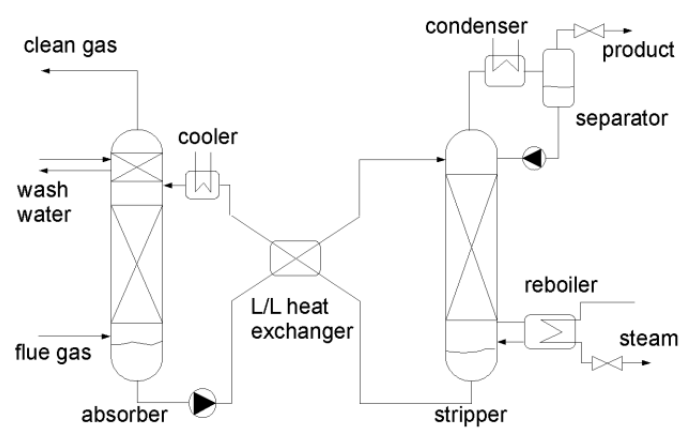

Figure 1: Schematic of an absorption/desorption process to remove carbon dioxide from power plant flue gas.

steam from the power generation process. Leaving the stripper at the top the product stream is after water separation compressed and stored. The overall power plant efficiency is expected to be reduced by at least $10 \%$, the solvent regeneration being responsible for more than half of this [1]. Minimizing the amount of steam required in the reboiler is therefore the task with highest priority in the optimization of this process.

With an increasing demand on the plant's flexible operation in the face of frequent load changes and an increased fraction of the generation capacity expected to come from renewables, dynamic simulation and optimization have become important tools to ensure an efficient incorporation of the carbon capture into the power generation. At the same time a trade-off must be found between efficiency losses and removal rate, possibly governed by time-varying economic boundary conditions.

This paper presents the preliminary results achieved within a larger project aiming at developing 
an optimization technology for advanced modelbased control of the separation plant. It focuses on the modeling of the capture plant, briefly presents the methods and tools that are used for optimization and presents preliminary results of solving an optimal control problem for the reduced model presented in the first half of the paper.

\section{Background}

\subsection{Modeling of carbon dioxide removal with chemical absorption}

System simulation models of amine scrubbing processes with different levels of detail can be found in the literature and as part of commercial toolboxes. The most rigorous models are developed for steadystate system computations with partial differential equations for mass transport along bulk flow and between the two phases, resulting in a high order system. This becomes easily too complex for dynamic system simulations, especially if parts of the power generation are supposed to be included or if used in model based control. Replacing rigorous models of multi-component mass transfer between gas and liquid with semi-empirical algebraic correlations reduces model complexity dramatically and is for example applied in [2] for an absorber description. Another model aspect with room for different levels of detail is the thermodynamic model of the liquid phase, describing the non-ideality of the electrolyte solution. Tobiesen compares in [3] a more rigorous with simpler approaches and concludes that high accuracy is rather a matter of a good data fit than model complexity.

Several studies on optimal operation of an aminebased $\mathrm{CO}_{2}$ capture plant can be found in the literature. In [4] the effect of variables such as solvent circulation rate, stripper pressure or solvent temperature is investigated. The analysis is however static and considered only the variation of one parameter at a time, disregarding the multivariable and dynamic nature of the process. In [5] control strategies aiming at a fast response are developed using offline dynamic simulation of the process. In [6], both optimization and control of the plant are studied. The optimal conditions for operation are determined offline using static models and a suitable control structure to maintain the process close to optimal operation in spite of disturbances is thereafter derived using dynamic models.

The process industry has up to now not taken up the use of Modelica to the same degree as e.g. the automotive industry, mainly due a strong market presence of domain-specific tools that are only applicable to process industry problems. Another important reason is the lack of physical properties for substances used in the process industry. There are, however, no other languages and tools that are as suitable as the combination of Dymola for highperformance simulation and JModelica.org for dynamic optimization for the given project, when the threshold of developing the fluid property models natively in Modelica is overcome.

\subsection{Model Predictive Control}

Model Predictive Control (MPC) is an advanced control method that relies of on-line solution of optimal control problems. During recent years, the method has become increasingly popular, especially in the process industry, [7]. The popularity of the method is attributed to its ability to handle multipleinput multiple-output (MIMO) systems, as well as control and state constraints. These two ingredients are common in a broad range of control problems. MPC allows the control engineer to tune a cost function to express the control objectives, typically by choosing weights in a quadratic cost function. By choosing the weights properly, the significance of the control objectives can be balanced. E.g., performance can be traded for robustness. In order to capture limitations in the plant to be controlled, constraints can be modeled. Constraints may represent tanks that may not over-flow or pressures that may not be exceeded for safety reasons. Other examples of constraints include limitations in actuators, such as limited ranges in valves and limited torques in motors.

In addition to a cost function and constraints, MPC relies on a model of the plant to be controlled. The model may be derived from first-principles, as is the case in this paper, or it may be computed from empirical data. Both linear and non-linear models can be used. During execution of the MPC controller, the model is used to predict the plant response to the future control inputs.

The key component of an MPC controller is the solution of an open loop optimal control problem (OCP). Based on the cost function, the constraints, the model and measurements, or estimates of the current plant state, optimal predicted trajectories for the model variables and the control inputs are computed. The first part of the optimal control variable trajectories is then applied to the plant. The procedure is then repeated periodically, each time shifting the optimal control horizon one step into further. This principle is called receding horizon control.

Solution of optimal control problems may be very computationally challenging, in particular for nonlinear models. Application of MPC is therefore more 
common in domains where typical plants have time constants in the range of minutes and hours rather than seconds. The CCS systems studied in this paper falls into this category, which makes MPC a feasible choice.

In addition to industrial use, MPC has also been extensively studied in the academic community, where a large body of theory has been developed, see, e.g., [11,12]. Notably, results for optimality, stability and robustness are available.

\subsection{JModelica.org, Optimica and Dymola}

In this work, Dymola is used as platform for simulation and as graphical editor while the software platform JModelica.org is used to solve dynamic optimization problems is JModelica.org. The JModelica.org platform has been described earlier [8], and is currently undergoing rapid development both with respect to the parts of the Modelica language that are supported and with respect to the algorithms available. The main reason for choosing the JModelica.org platform is, however, that it offers strong support for solution of dynamic optimization problems, which is a key component of executing MPC controllers, as discussed above.

JModelica.org supports an extension of Modelica entitled Optimica [9], which allows dynamic optimization problems to be formulated based on Modelica models. Optimica enables the user to express cost functions, constraints, and what to optimize in a description format that is complimentary to Modelica's support for dynamic modeling using high-level language constructs. This feature enables shorter design cycles since more effort can be put into formulation of optimization problems rather than encoding them in a specialized format for a particular optimization algorithm. This property is valuable in this this work, since extensive tuning of the cost functions and the constraints has proven necessary.

A direct collocation method, [10], is implemented in JModelica.org for solving large scale dynamic optimization algorithms. The method is applicable to differential algebraic systems and relies on full discretization of state, algebraic and control profiles. The resulting non-linear program is typically very large, but also sparse, which can be exploited by numerical software. In JModelica.org, the algorithm IPOPT, [11], is used to solve the NLPs resulting from collocation.

In terms of user interaction, JModelica.org offers a Python [12] interface. Using Python, Modelica and Optimica models can be compiled into executable optimization programs, optimization algorithms can be invoked and the results can be loaded. Python also comes with packages for numerical computations and visualization, which makes it a suitable environment for scientific computations. It can be noted that the capabilities of Python go beyond scripting and atomization in that full-fledged applications with customized user interfaces can be created.

\section{Dynamic model of an absorption/desorption column}

The starting point in the development of a Modelica model suitable to be used in dynamic optimization is a model of an absorption unit developed in Dymola. The system consists of the main components absorber, stripper, reboiler and internal heat exchanger as well as auxiliary equipment such as pumps, valves, flow resistances, cooled vessels, sensors and reservoirs, as sketched in Figure 1. The solvent is an aqueous MEA solution.

Each packed section in a column consists of gas and liquid bulk flow and a static interface model describing the two-phase contact. Figure 2 shows the diagram layer of the packed section model. Gas and liquid phase are treated as separate media, each modeled as a separate medium property package. Thermodynamic equilibrium is only present at the phase interface, while mass and energy storage only occurs in the bulk flow.

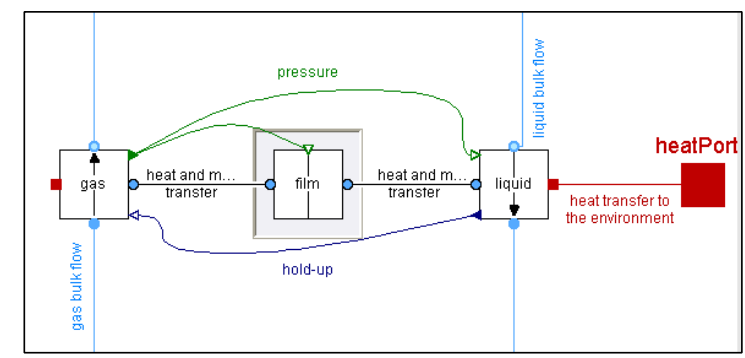

Figure 2: Diagram of the packed section model

Phase equilibrium at the gas-liquid interface for both, water and carbon dioxide, is computed as follows, assuming the pointing-factors and gas phase fugacity coefficients being equal to one.

$$
\begin{aligned}
& y_{\mathrm{CO} 2} p=\gamma_{\mathrm{CO} 2} x_{\mathrm{CO} 2} \mathrm{He}_{\mathrm{CO} 2} \\
& y_{\mathrm{H} 2 \mathrm{O}} p=\gamma_{\mathrm{H} 2 \mathrm{O}} x_{\mathrm{H} 2 \mathrm{O}} p_{\mathrm{H} 2 \mathrm{O}, \mathrm{sat}}(T)
\end{aligned}
$$

with the mole fractions in gas and liquid phase $y_{i}$ and $x_{i}$, the Henry-coefficient for dissolution of $\mathrm{CO}_{2}$ in water $\mathrm{He}$, the vapor pressure of water $p_{i, s a t}$ and the system pressure $p$.

\subsection{State selection}

Pressure in the column is determined by the gas phase, with friction losses along the way through the packing material. The space available for the gas 
phase is however dependent on the space occupied by the liquid phase. These properties and their derivatives are then passed to the respective other bulk component through signal connectors, see green and dark blue connections in Temperature and species amounts in each phase were chosen as independent state variables. Algebraic loops and high index problems can thus be avoided if

1. gas pressure can be directly computed from temperature and species amounts in the gas phase, e.g. using the ideal gas law or a cubic equation of state,

2. liquid density is independent of pressure (incompressible medium),

3. energy and species mass balances are formulated in terms of the derivatives of the chosen states,

4. and mass and heat transfer correlate concentrations and temperatures in the two dynamic volume models, gas and liquid bulk flow

Pressure drop in the gas phase and liquid hold-up are determined with literature correlations for packed columns, e.g. [Mackowiak], or user-defined nominal points, i.e. constant hold-up and gas flow operating point. The actual liquid hold-up correlates with the static set point via first order dynamics.

For a stripper column operated with MEAsolution and under the assumption that MEA is nonvolatile, the number of dynamic degrees of freedom is then equal to 7 per volume segment $\left(\mathrm{CO}_{2}\right.$ gas, $\mathrm{H}_{2} \mathrm{O}$ gas, $\mathrm{CO}_{2}$ liquid, $\mathrm{H}_{2} \mathrm{O}$ liquid, MEA, T liquid, $\mathrm{T}$ gas). In the absorber absorber additional flue gas component as oxygen and nytrogen are present. Column design, operation and demanded accuracy determine the required discretization of the packed sections in bulk flow direction, which usually is a number between 8 and 20.

\subsection{Chemical reactions}

The capacity of amines to absorb carbon dioxide is to a large extent based on chemical reactions. In the case of MEA as a solvent five main reactions can be identified as well as the zero charge condition.

$$
\begin{aligned}
& 2 \mathrm{H}_{2} \mathrm{O} \leftrightarrow \mathrm{H}_{3} \mathrm{O}^{+}+\mathrm{OH}^{-} \\
& \mathrm{CO}_{2}+2 \mathrm{H}_{2} \mathrm{O} \leftrightarrow \mathrm{H}_{3} \mathrm{O}^{+}+\mathrm{HCO}_{3}^{-} \\
& \mathrm{HCO}_{3}{ }^{-}+\mathrm{H}_{2} \mathrm{O} \leftrightarrow \mathrm{H}_{3} \mathrm{O}^{+}+\mathrm{CO}_{3}{ }^{2-} \\
& \mathrm{MEAH}^{+} \mathrm{H}_{2} \mathrm{O} \leftrightarrow \mathrm{H}_{3} \mathrm{O}^{+}+\mathrm{MEA} \\
& \mathrm{MEACOO}^{-}+\mathrm{H}_{2} \mathrm{O} \leftrightarrow \mathrm{MEA}+\mathrm{HCO}_{3}{ }^{-}
\end{aligned}
$$

This leads to a total of nine species in the liquid phase including the 6 ions. Throughout the models developed within this work, chemical equilibrium is assumed to be present, at the phase interface as well as in the bulk liquid. This assumption is thought to be justified at high temperatures as they are found in the stripper. The deviations resulting in the absorber are considered acceptable, if taking into account the poor availability of reliable kinetic data in the literature and the amount of additional dynamic states saved (5 per volume segment). However, a different solvent may demand a different approach.

\subsection{Chemical equilibrium}

The liquid phase speciation is determined by equilibrium constants $K_{\mathrm{j}}$ from the literature for each reaction $\mathrm{j}$, which are determined empirically and expressed as polynomial functions of temperature. They are defined as

$$
K_{\mathrm{j}}=\prod\left(\gamma_{i} m_{i}\right)^{v_{i, j}}
$$

where $\gamma_{i}$ and $m_{i}$ are the activity coefficient and molality of component $\mathrm{i}$, respectively. $v_{i, j}$ is the stoichiometric coefficient of component $i$ in reaction $j$, starting materials are considered with a negative sign, products with a positive one. Equilibrium constants allow also for an inference on heats of reaction, using the van't Hoff equation:

$$
\frac{d \ln K}{d T}=\frac{\Delta H_{r}}{R T^{2}}
$$

where $\Delta H_{r}$ is the enthalpy of reaction, $T$ the temperature and $R$ the ideal gas constant. The enthalpy of physical solution is computed accordingly using the temperature dependency of the Henry-coefficient [13].

However, a lot of computational time is required to solve the non-linear system of equations describing the speciation. Furthermore, extreme differences in ion concentrations by several orders of magnitude make a good choice of iteration variables essential for robust convergence.

In addition the following assumptions also apply:

- the flue gas entering the absorber contains only carbon dioxide, water, oxygen and nitrogen

- MEA is non-volatile and not present in the gas phase

- the total amount of liquid in the column is defined as the packing hold-up and the sump liquid volume 
- the liquid in the column sumps and other large volumes is assumed to be ideally mixed

- mass and heat transfer between liquid and gas phase is restricted to the packed section

- negligible temperature difference between liquid bulk and interface to gas phase

- perfect gas law applies in the gas phase.

- phase equilibrium in reboiler and condenser

Table 1: References for physical properties used in the model

\begin{tabular}{|l|l|l|l|}
\hline Property & Symbol & $\begin{array}{l}\text { Used in } \\
\text { reduced } \\
\text { model }\end{array}$ & Reference \\
\hline $\begin{array}{l}\text { Equilibrium } \\
\text { constants }\end{array}$ & $K_{\mathrm{i}}$ & indirectly & $\begin{array}{l}\text { Collected } \\
\text { in [14] }\end{array}$ \\
\hline $\begin{array}{l}\text { Henry- } \\
\text { coefficient }\end{array}$ & $H e_{\mathrm{co} 2}$ & yes & {$[14]$} \\
\hline $\begin{array}{l}\text { Activity } \\
\text { coefficients, } \\
\text { liquid phase }\end{array}$ & $\gamma_{\mathrm{i}}$ & indirectly & {$[14]$} \\
\hline $\begin{array}{l}\text { Mass transfer } \\
\text { coefficients }\end{array}$ & $k_{\mathrm{iL}}, k_{\mathrm{iv}}$ & no & {$[15]$} \\
\hline $\begin{array}{l}\text { Diffusivities } \\
\text { liquid phase }\end{array}$ & $D_{\mathrm{iL}}$ & no & $\begin{array}{l}{[16]+} \\
\text { Stokes - } \\
\text { Einstein } \\
\text { relation }\end{array}$ \\
\hline $\begin{array}{l}\text { Diffusivities } \\
\text { gas phase }\end{array}$ & $D_{\mathrm{iV}}$ & no & $\begin{array}{l}\text { Fuller's } \\
\text { eq.in [17] }\end{array}$ \\
\hline $\begin{array}{l}\text { Densities and } \\
\text { viscosities, } \\
\text { liquid }\end{array}$ & $\rho, \mu$ & yes & {$[18]$} \\
\hline $\begin{array}{l}\text { Enhancement } \\
\text { factor }\end{array}$ & $E$ & no & {$[2]$} \\
\hline
\end{tabular}

The molecular carbon dioxide concentration $\mathrm{c}_{\mathrm{CO} 2, \mathrm{~b}}$ is then used to compute mass transfer between bulk and interface (if).

$\dot{n}_{i L}=A_{i f} k_{i L} E\left(c_{i, b}-c_{i, i f}\right) \mathrm{i}=\mathrm{CO}_{2}$ $\dot{n}_{i V}=\frac{A_{i f} k_{i V}\left(p_{i, b}-p_{i, i f}\right)}{R T} \mathrm{i}=\mathrm{CO}_{2}, \mathrm{H}_{2} \mathrm{O}$

where $\dot{n}_{i L}$ and $\dot{n}_{i V}$ denote the molar flows in the liquid and the vapor phase, respectively. $A_{i f}$ is the contact area, $E$ is an enhancement factor describing the impact of chemical reactions on the concentration profile near the interface. $k$ is a mass transfer coefficient, $c_{i, i f}$ and $c_{i, b}$ are molar concentrations at the interface and in the liquid bulk, respectively and $p_{i, i f}$ and $p_{i, b}$ are correspondingly partial pressures of the considered species in the gas phase. $R$ and $T$ are the ideal gas constant and bulk phase temperature, respectively.

Properties and correlations from the literature used in these models are listed in Table 1.

\subsection{Model reduction}

Online optimization as it is used in MPC implicates tighter limitations on the model size than pure dynamic simulation or even offline optimization would do. The solution of the optimization problem for a finite horizon needs to be found between two sampling instants and therefore demands a relatively low computational effort. But also the available memory to store result points for all model variables for each time step within the finite horizon limits the allowed number of algebraic and differentiated variables. However, exact numbers are hard to define in advance. At the same time accuracy demands are not as high as the model is updated with measurement values at each sample step.

The following measures are taken in order to reduce the model:

1. Chemical equilibrium computation (and ion speciation) was replaced by a spline approximation of the molecular $\mathrm{CO}_{2}$ concentration in the liquid phase as a function of temperature and solvent loading with $\mathrm{CO}_{2}$. The mass fraction of MEA in the unloaded solution is kept constant at $30 \%$ for this function. 
2. Enthalpy of absorption/desorption is replaced by a function of temperature but constant with solvent loading.

3. Mass transfer coefficients including enhancement by chemical reactions are no longer computed from physical medium properties, but become constant tuning parameters.

4. Reduction of the number of volumes in bulk flow direction to an acceptable minimum (iterative, dependent on application)

5. Constant specific heat capacities of all species and constant liquid density

\subsection{Validation and model comparison}

The total system model is composed of the two packed columns and complemented with washers, condensers, pumps and valves according to Figure 1. The reboiler, which supplies the gas flow to the stripper is modeled as a flash stage with phase equilibrium and uniform temperature. Simulation results of the detailed model are compared to experimental data from a pilot plant run with open control loops [19]. The input variables of the test case are:

- fluegas inlet flow and properties

- clean gas pressure

- liquid recirculation rate

- reboiler duty

- product stream outlet pressure

All inlet conditions are kept constant except for the flue gas rate, which is reduced by $30 \%$ after having run the plant in steady-state for some time. Figure 3

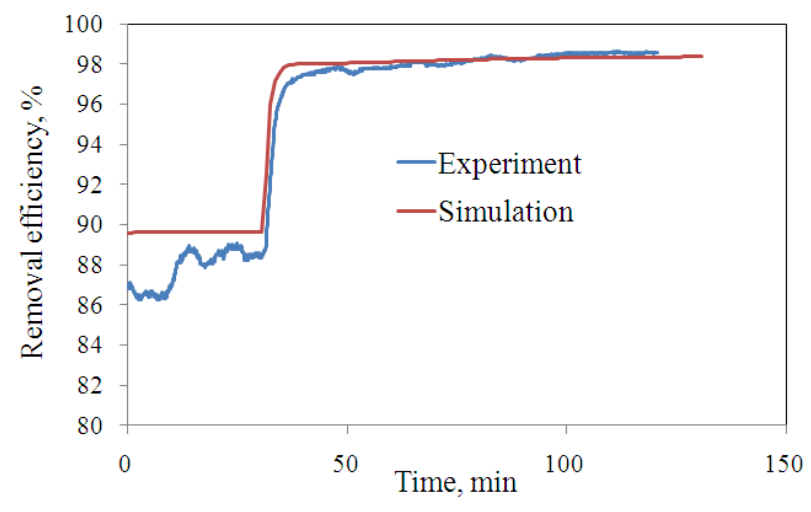

Figure 3: Carbon dioxide removal rate, experiment and simulation of the detailed model

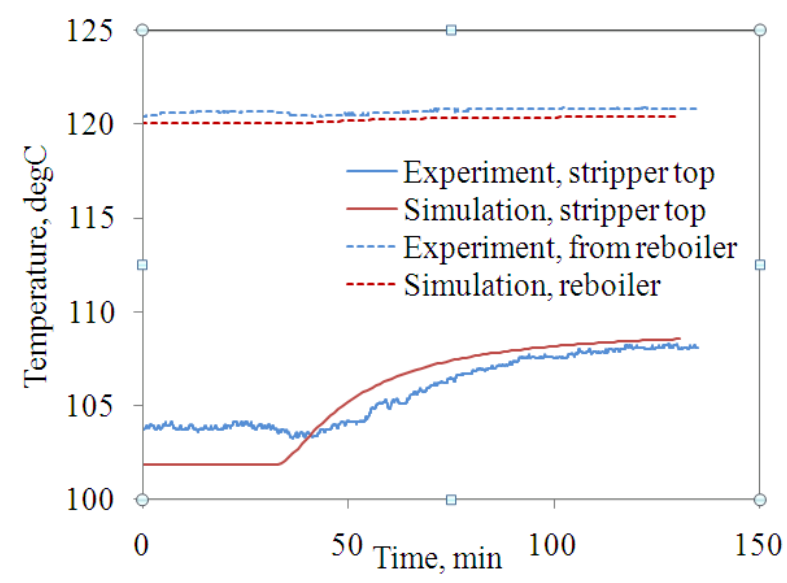

Figure 4: Stripper top and reboiler temperatures

shows the $\mathrm{CO}_{2}$ removal rate before and after the step change in experiment and simulation. Giving the fact that the experiment apparently did not reach steadystate before the step, the agreement between the two curves is satisfactory.

The temperatures at the gas outlet of the stripper column and at the liquid outlet of the reboiler are plotted in Figure 4.

Especially the reboiler temperature, which is directly coupled to pressure and pressure drop along the gas flow path as well as the solvent loading, is in very good agreement with the experimental data.

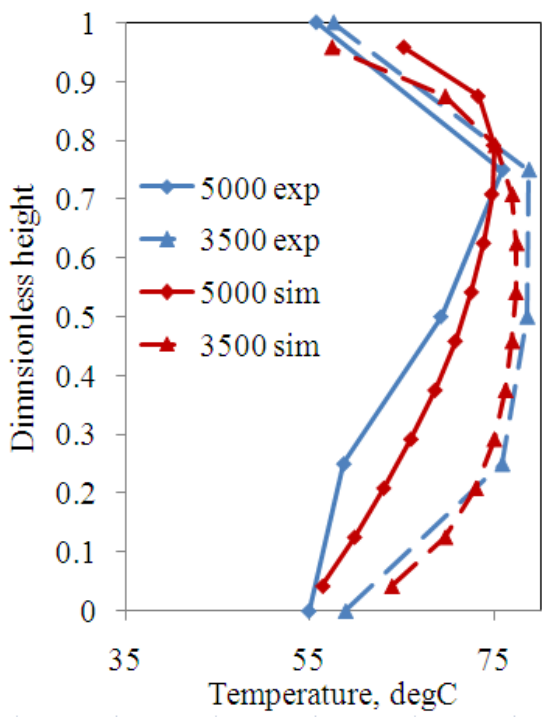

Figure 5: Temperature profile wrt column height

Since liquid phase concentration data is unavailable, it can be useful to look at temperatures instead, because of the direct connection between ab- 
sorbed/desorbed carbon dioxide and temperature changes due to heats of reaction. Figure 5 compares the gas temperature profile along the absorber column height for experiment and simulation at presumed steady state before and after the flue gas step, respectively. The locations of the five measurement points were guessed to be equally distributed. The simulation captures well the location of the highest temperature first in the upper part and later with a lower gas flowrate as having moved further down.

The optimization problem in the next section is only solved for the stripper column including reboiler and condenser. A comparison of the detailed and the reduced model is therefore only performed for this part of the plant. Model assumptions, which affect the dynamic behavior of the unit, namely concerning liquid volumes and hold-ups, are similar in both models. Therefore, the comparison is restricted to steady-state operating points. Figure 6 presents the liquid lean loading at the stripper outlet as a result of reboiler duty under constant liquid inlet conditions and stripper top pressure. The results show that the energy required to regenerate the solvent to a certainn lean loading is predicted close to each other with the two models. It can be concluded that the complexity of the reduced model is sufficient to investigate the energy consumption of the reboiler. The reduced model performed the stripper unit series 200 times faster than the detailed model. The simulations started at fixed initial states and simulated to steadystate. Large transients as they occur in the first seconds of a simulation demand especially large computational efforts, when using the detailed model.

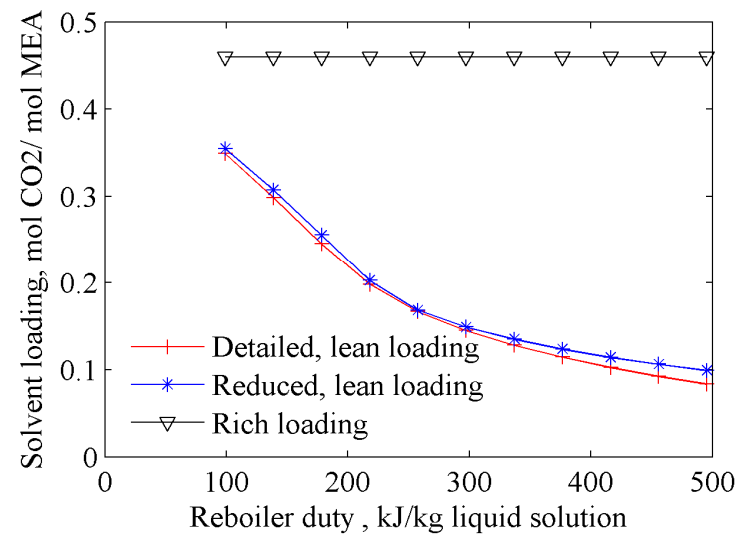

Figure 6: Solvent lean loading as a function of reboiler duty, detailed and reduced model

\section{Optimization results}

The goal of the project is to apply nonlinear model predictive control on the separation plant to minimize its energy usage. As it was mentioned in Section 2.2 , this requires solving a sequence of open-loop optimal control problems. The aim of the present section is to show how those open-loop control problems can efficiently and accurately be solved using the simplified models and the tools previously described. For that purpose, a simple control problem using one of the most energy demanding parts of the separation plant, namely the stripper unit, will be formulated and solved.

\subsection{Process model}

The process to be optimized is the stripper unit shown in Figure 7.

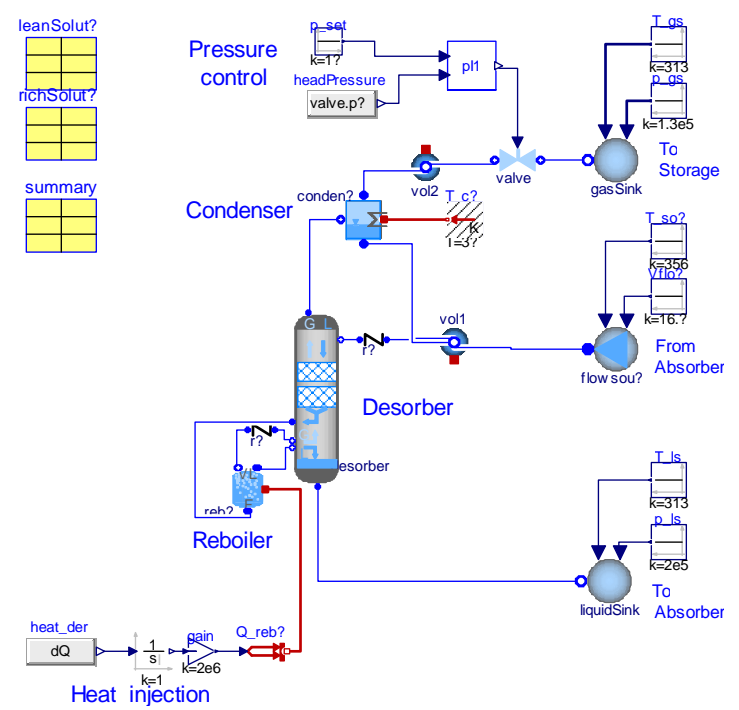

Figure 7: Graphical representation of the stripper unit used for optimization in Dymola

It is composed of:

- a reboiler

- a stripper column with packed sections and a sump

- a condenser to remove the water from the product stream

- a pressure control valve together with a pressure controller 
The process model is described by 1493 equations and 1493 time-varying variables, including 50 continuous-time states. This is a larger model size than the size of the models reported in [20] for start-up optimization of coal fired power plants.

\subsection{Control problem}

Objective function. The control problem is formulated as in standard MPC using a quadratic cost function $J$ penalizing deviations of the controlled variable $y$, as well as variations in the control signal $u$ :

$$
J\left(u, x_{0}\right)=\int_{0}^{H_{p}} \alpha\left(y(t)-y_{r e f}\right)^{2}+\beta\left(\frac{d u}{d t}\right)^{2} d t
$$

where $\alpha$ and $\beta$ are weights that can be tuned to achieve a desired dynamic behavior and $H_{p}$ is the prediction horizon.

Controlled variable. The variable to be controlled is the removal efficiency $\eta$ of the separation plant. It is defined as the mass flow ratio of carbon dioxide leaving the condenser and carbon dioxide entering the absorber column with the fluegas:

$$
\eta=\frac{\dot{\mathrm{m}}_{\mathrm{CO} 2, \text { condenser out }}}{\dot{\mathrm{m}}_{\mathrm{CO} 2, \text { absorber in }}}
$$

Since the absorber column is not included in the optimization set-up, the $\mathrm{CO}_{2}$ concentration in the rich solution entering the stripper column has been assumed to be in equilibrium with the flue gas entering the absorber column.

Control signal. The chosen control signal is the heat flow rate $\dot{Q}$ to the reboiler. However, the decision variable of the optimization problem is chosen to be its time-derivative $\frac{d \dot{Q}}{d t}$, which is parameterized by a piecewise constant signal taking $\mathrm{N}$ values over the prediction horizon $H_{p}$, i.e. for $\mathrm{i}=0 . . \mathrm{N}-1$

$$
\frac{d \dot{Q}}{d t}(\mathrm{t})=\gamma_{i} \in R, t \in\left[i \frac{H_{p}}{N},(i+1) \frac{H_{p}}{N}\right]
$$

Only the first value of this open-loop optimization result, i.e. $\gamma_{0}$ would actually be applied to the process if the entire MPC algorithm was implemented.

Constraints. As far as the optimization constraints are concerned, they may be of both regulatory ${ }^{1}$ and operational nature. The versatile JModelica.org plat-

\footnotetext{
${ }^{1}$ Regulatory rules for carbon capture plants are still under discussion, but will certainly play a role.
}

form allows us to include any constraint that can be expressed in terms of process variables. In the present example, an upper limit on the reboiler pressure is imposed to avoid MEA degradation occurring at high temperatures.

$$
p_{\text {reboiler }}(t) \leq p_{\max }, \quad t \in\left[0, H_{p}\right]
$$

A maximal temperature could equivalently be imposed since pressure and temperature are coupled in the reboiler.

Initial state. The initial state $x_{0}$ is assumed to be known and is computed using Dymola as the stationary point corresponding to a given heat flow rate $\dot{Q}=\dot{Q}_{0}$. An implementation of the MPC controller would require an observer to compute an estimate of the initial state $x_{0}$ based on the available measurements.

\subsection{Numerical example}

As mentioned in Section 2.3, the JModelica.org platform implements a direct collocation method to solve the optimal control problem. This implies that optimization is not performed on the continuous DAE system mentioned in 4.1, but on a discretized version using the Radau quadrature. The trajectory of every variable in the dynamic model is approximated by piecewise polynomials on each interval of the prediction horizon. In each interval, the approximation is exact at a number $N_{c}$ of points, the collocation points. Choosing $N_{c}=3$ and dividing the prediction horizon $H_{p}$ in $\mathrm{N}=10$ intervals of equal length converts the continuous optimization problem to an algebraic nonlinear program with 29824 variables, 29814 equality constraints and 5646 inequality constraints. Most of the inequality constraints originate from the max and min attributes associated to the physical variables. As the optimization problem is most probably non-convex, it is essential to provide the solver IPOPT with reasonable guessed trajectories for the initialization of the iterative optimization algorithm. The trajectories were here taken to be constant in time and given by the initial state $x_{0}$, computed in Dymola.

A step change in the desired removal efficiency is now considered. Using the numerical values listed in Table 2, the optimization problem is solved in JModelica.org in 36 iterations. The results are shown in Figure 8. 
Table 2: Parameter values used in the optimization problem

\begin{tabular}{|l|l|l|l|l|l|}
\hline & & & & & \\
\hline $1000 \mathrm{~s}$ & 0.1 & & $0.7 \mathrm{M}$ & $\begin{array}{l}1.95 \\
\text { bar }\end{array}$ & 0.9 \\
\hline
\end{tabular}

At the beginning, the heat flow rate to the reboiler is rapidly increased from its start value of $0.7 \mathrm{MW}$ to $1.05 \mathrm{MW}$, leading to a removal efficiency of about 0.8 at time $\mathrm{t}=400 \mathrm{~s}$. At around $500 \mathrm{~s}$, the reboiler pressure reaches its maximal allowed value of 1.95 bar and the heat flow rate decreases slightly to avoid constraint violation. Because of the high condenser pressure, the target efficiency of 0.9 cannot be achieved in this optimization setup. With a different column design or different boundary conditions, higher efficiency could of course be achieved.

To evaluate the consistency of the optimization result with respect to the continuous-time model equations, the optimized trajectories have been evaluated by applying the optimized heat input to the model implemented in Dymola. No difference could be observed when comparing results from JModelica.org and Dymola (results not shown).
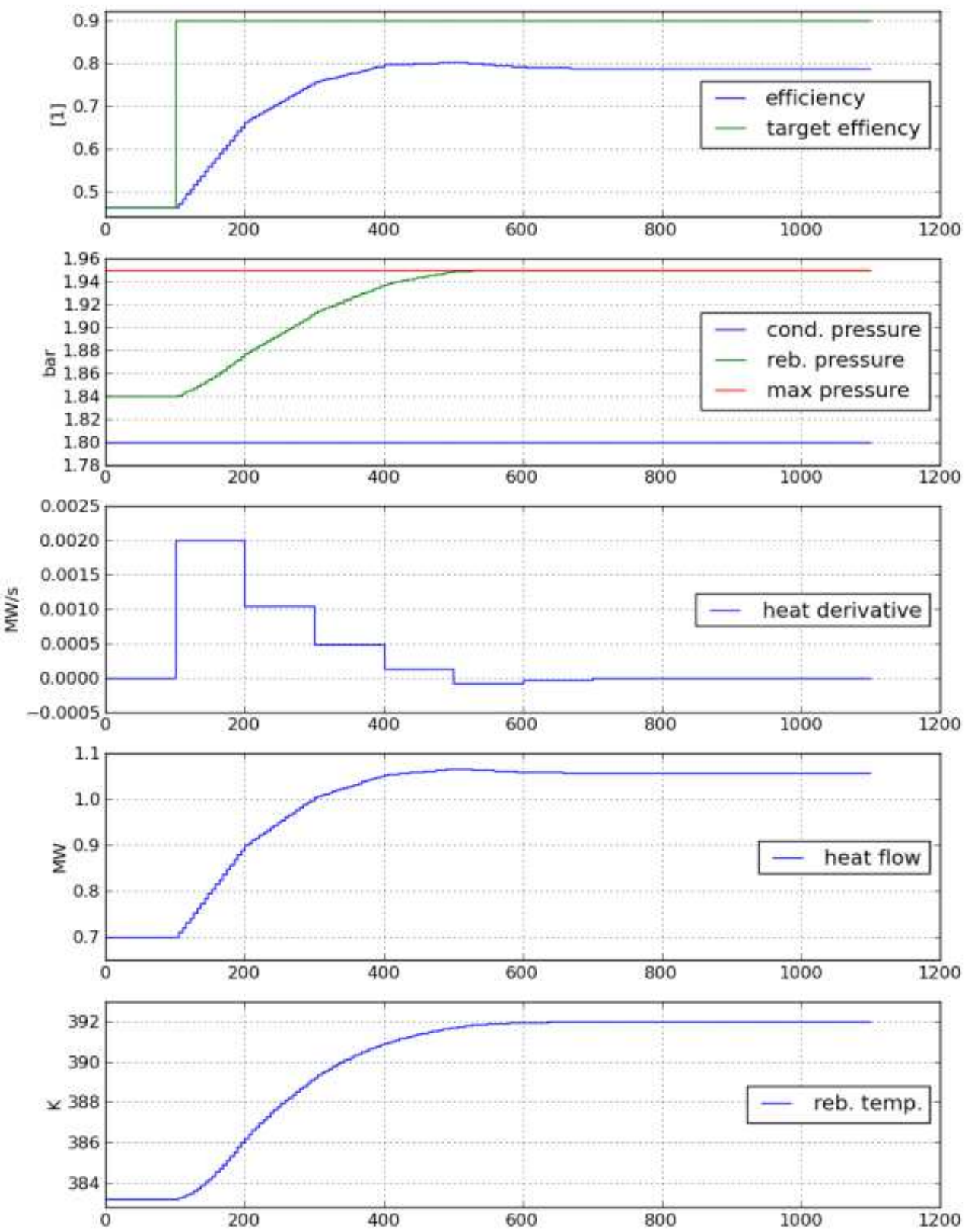


\section{Conclusions}

A dynamic model of a post-combustion carbon capture process developed in Modelica was presented. The main focus lies on the chemical absorption of the carbon dioxide by the liquid solution in the absorber column. The same model can be used for the corresponding desorption process in the stripper column, by exchanging the flue gas medium for a mixture of water steam and carbon dioxide. A comparison of simulation results with experiments from a pilot plant showed a good agreement.

In a second step the model was reduced to meet the demands of a dynamic optimization. The largest performance improvement was achieved with a replacement of the chemical reactions in the liquid phase by an interpolated table with equilibrium data. A comparison of steady-state results from the stripper unit modeled with both approaches justified the usage of the reduced model for energy optimization purposes.

As a first step toward NMPC, a test case with the chosen system model was defined. It demonstrates the solution of an optimal control problem with the JModelica.org platform while adhering to specified variable constraints, in this case set on the reboiler pressure.

By formulating and solving this problem we have shown that the JModelica.org platform is a viable choice for solving large scale dynamic optimization problems, which is a prerequisite for NMPC applied to CCS plants. Future extensions include investigation of how to explore available control variables, cost function formulation, and state estimation.

\section{Acknowledgements}

This work was funded by the Swedish funding agency Vinnova under the grant program "Forska and Väx.

\section{References}

[1] Abu-Zahra MRM, Schneiders LHJ, Niederer JPM, Feron PHM, Versteeg GF. CO2 capture from power plants. Part II. A parametric study of the economical performance based on mono- ethanolamine. International Journal of Greenhouse Gas Control 2007;1:135-142.

[2] Kvamsdal HM, Jakobsen JP, Hoff KA. Dynamic modeling and simulation of a $\mathrm{CO}_{2}$ absorber column for post-combustion $\mathrm{CO}_{2}$ capture. Chem Eng Process 2009;48:135-144.

[3] Tobiesen FA, Juliussen O, Svendsen HF. Experimental validation of a rigorous stripper modelfor $\mathrm{CO}_{2}$ post-combustion capture. Chem Eng Sci 2008;63:2641-2656.

[4] Freguia S, Rochelle GT. Modeling of CO2 capture by aqueous monoethanolamine. AIChE Journal 2003;49(7):1676-1686.

[5] Ziaii S, Rochelle GT, Edgar TF. Dynamic modeling to minimize energy use for $\mathrm{CO} 2$ capture in power plants by aqueous monoethanolamine. Ind Eng Chem Res 2009;48:6105-6111.

[6] Panahi M, Karimi M, Skogestad S, Hillestad M, Svendsen HF. Self-optimizing and control structure design for $\mathrm{a} \mathrm{CO}_{2}$ capturing plant. In: Eljack FT, Rex Reklaitis GV, El-Hawagi MM, editors. Proceedings of the 2nd Annual Gas Processing Symposium, Qatar 2010.

[7] Qin SJ, Badgwell TA. A survey of industrial model predictive control technology. Control Engineering Practice 2003;11: 733-764.

[8] Åkesson, J., Årzén, K.-E., Gäfvert, M., Bergdahl, T., \& Tummescheit, H. Modeling and Optimization with Optimica and JModelica.org_-Languages and Tools for Solving Large-Scale Dynamic Optimization Problem. Computers and Chemical Engineering 2010, Doi:10.1016/j.compchemeng.2009.11.011.

[9] Åkesson, J. Optimica-an extension of Modelica supporting dynamic optimization. 6th International Modelica Conference 2008.

[1] Biegler LT, Cervantes A, Wächter A. Advances in simultaneous strategies for dynamic optimization. Chemical Engineering Science 2002;57:575-593.

[11] Wächter A, Biegler LT. On the implementation of an interior-point filter line-search algorithm for large-scale nonlinear programming. Mathematical Programming , 2006;106 (1); 2558.

[12] Python Software Foundation. Python Home Page. Retrieved from www.python.org, 2010.

[13] Kim I, Hesssen ET, Haug-Warberg T, Svendsen HF. Enthalpies of Absorption of $\mathrm{CO}_{2}$ in Aqueous Alkanolamine Solutions from e-NRTL Model. Energy Procedia 2009;1:829-835.

[14] Böttinger W. NMR-spektroskopische Untersuchung der Reaktivabsorption von 
Kohlendioxid in wässrigen Aminlösungen. Dissertation. Universität Stuttgart; 2005

[15] Onda K, Takeuchi H, Okumoto Y. Mass transfer coefficients between gas and liquid packed columns J Chem Eng Jpn 1968;1(1):56-62

[16] Versteeg GF, van Dijck LAJ, van Swaaij WPM. On the kinetics between $\mathrm{CO}_{2}$ and alkanolamines both in aqueous and non-aqueous solutions. An overwiew. Chem Eng Commun 1996;144:113158.

[17] Poling BE, Prausnitz JM, O'Connell JP. The properties of gases and liquids. 5th edition. New York: McGraw-Hill;2001

[18] Weiland RH, Dingmann JC, Cronin DB, Browning GJ. Density and Viscosity of Some Partially Carbonated Aqueous Alkanolamine Solutions and Their Blends. J. Chem. Eng. Data 1998;43(3):378-382

[19] Faber R, Köpcke M, Biede O, NygaardKnudsen J, Andersen J. Open-loop step responses for the MEA post-combustion capture process: Experimental results from the Esbjerg pilot plant. Proceedings of the GHGT-10 conference 2010.

[20] Franke, R. Doppelhamer, J., Online Application of Modelica Models in the Industrial IT Extended Automation System 800xA, Proceedings of 5th International Modelica Conference, 2006, Vienna, Austria. 